\title{
Role of the conserved charges in the chiral symmetry restoration phase transition
}

\author{
Pedro Costa๑, ${ }^{*}$ Renan Câmara Pereira $\odot,{ }^{\dagger}$ and Constança Providência $\odot^{\ddagger}$ \\ CFisUC, Department of Physics, University of Coimbra, P-3004-516 Coimbra, Portugal
}

(Received 3 April 2020; accepted 30 July 2020; published 11 September 2020)

\begin{abstract}
The effect of conserved baryon, isospin, and strangeness charges on the behavior of phase transitions in dense matter is studied. Baryonic matter is described within the three-flavor Polyakov-Nambu-JonaLasinio model and several charge fractions $Y_{Q}$ are considered. The role of the vector interaction, which can be important to describe dense systems, is discussed. Special attention is given to the case with charge fraction $Y_{Q}=0.4$, due to its importance in heavy-ion collisions and core-collapse supernova matter. It is shown that the possible formation of chiral-symmetric quark matter in the laboratory will be favored in asymmetric matter. Besides, the inclusion of the vector interaction reinforces the formation of quark matter at lower densities.
\end{abstract}

DOI: $10.1103 /$ PhysRevD.102.054010

\section{INTRODUCTION}

We investigate the phase transition associated with the restoration of chiral symmetry in a system with more than one conserved charge (multicomponent systems): baryonic charge, isospin, and strangeness. This is relevant for scenarios where asymmetric matter occurs, like in heavyion collisions (HICs) [1] and compact stars [2]. Given a fixed electric-to-baryon charge ratio, $Q / B \neq 0.5$, this additional isospin degree of freedom cannot be exploited by the system in pure phases. In the mixed phase, however, the total asymmetry is constant, while the local asymmetries of each phase can be different. For more than one globally conserved charge, such as baryon, isospin, and/or strangeness, phase equilibrium has to be implemented by imposing Gibbs rules, which modify both the structure of the mixed phase and the determination of the transition point. When only one globally conserved charge is allowed, the phase equilibrium is obtained by a Maxwell construction at constant pressure.

In the modelling of a first-order phase transition with density as the order parameter, it is often assumed that the coexistence region can be obtained by a Maxwell construction at constant pressure. However, this is not true if different charges correspond to good quantum numbers, and in that case full Gibbs conditions must be applied [3].

\footnotetext{
*pcosta@uc.pt

renan.pereira@student.uc.pt

*cp@fis.uc.pt
}

Published by the American Physical Society under the terms of the Creative Commons Attribution 4.0 International license. Further distribution of this work must maintain attribution to the author(s) and the published article's title, journal citation, and DOI. Funded by SCOAP ${ }^{3}$.
In a multicomponent system, the local concentrations of charges vary during the crossing of a phase-coexistence region, as well as the pressure and baryonic chemical potential. Considering the phase diagram in the temperature-pressure plane, for a given temperature, the transition occurs over a range of pressures. This effect is well known in plasma and condensed matter physics and was recently applied to the hadronic sector in Ref. [4].

In Ref. [5] the liquid gas phase transition in asymmetric nuclear matter, a system with more than one conserved charge, was intensely discussed. In fact, when building the equation of state for a neutron star, both electric and baryonic charges have to be conserved. The description of the phase transition from hadrons to quarks inside such an object is obtained by applying a Gibbs construction in order to identify the limits of the mixed phase [2]. Finding the equilibrium points that satisfy all Gibbs conditions may be cumbersome, but in Ref. [6] a new statistical method was introduced to study the thermodynamics of a multifluid system that reduced the problem to Maxwell constructions. It consists of keeping only one density fixed and replacing the others by their intensive conjugated variables.

We will perform our study within the $(2+1)$-flavor Polyakov-loop-extended Nambu-Jona-Lasinio (PNJL) model [7-11]. Models of the Nambu-Jona-Lasinio type are effective field theories describing the basic mechanisms that drive the spontaneous breaking of chiral symmetry [12] — a key feature of quantum chromodynamics (QCD) and are widely used to study the phase diagram of strongly interacting matter (also called the QCD phase diagram), hadron phenomenology, and the quark phase of the neutron star equation of state. The inclusion of the Polyakov loop in these models allows to better reproduce lattice-QCD results and to study the confinement-deconfinement transition. 
We consider a model with both a vector-isoscalar and a vector-isovector contribution, with equal coupling constants. Starting from a QCD-inspired color current-current interaction, these vector channels and the tensor channel can be related to the scalar-pseudoscalar channel using a Fierz transformation into color-singlet channels [13]. Indeed, in Refs. [14,15], a so-called Fierz-complete minimal set of channels was proposed from which any other interaction channel can be derived using Fierz transformations. In such an approach, the scalar-pseudoscalar and vector couplings are not independent and indeed they can be fixed by the scalar-pseudoscalar coupling $[13,16]$. From a phenomenological point of view, the vector coupling can also be fixed in the vacuum by requiring the model to be able to reproduce the vacuum masses of vector mesons. However, the vector interactions are known to couple to density degrees of freedom and the overall magnitude of the vector interaction might be density dependent, i.e., chemical potential dependent. In fact, there is still no constraint for the choice of an induced vector coupling at finite density. For example, we do not know if vector interactions induce a more attractive or repulsive interaction (and thus its sign is also unknown). Several studies of the QCD phase diagram and the neutron star equation of state have considered the vector couplings as free parameters (see, e.g., Refs. [9,17-23]):

(1) It has been shown that the vector interaction has a strong influence on the chiral-symmetry-restoration phase transition, making (for a strong enough interaction) a first-order phase transition turn into a smooth crossover.

(2) The vector channel is important for modeling the quark degrees of freedom inside the core of a neutron star [23].

In Refs. [24,25] the authors have shown that divergent density fluctuations result from spinodal decomposition in a nonequilibrium first-order chiral phase transition; in particular, the specific heat and charge susceptibilities diverge at the isothermal spinodal lines. The study was performed within the NJL model but the same conclusions are expected to be generally true. Understanding how the charge asymmetry may affect the metastable and unstable regions of the phase diagram, where this divergent behavior is also expected, is one of the objectives of our work.

This paper is organized as follows. In Sec. II the PNJL model, which will be used to model dense baryonic matter, is introduced. In Sec. III the results of considering more then one conserved charge in the PNJL model are presented and the phase transitions for different scenarios are shown. Finally, in Sec. IV we present out conclusions and describe some perspectives for future work.

\section{MODEL AND FORMALISM}

The $(2+1)$-flavor Lagrangian density for the PNJL model reads

$$
\begin{aligned}
\mathcal{L}= & \bar{\psi}\left(i \not D-\hat{m}+\hat{\mu} \gamma^{0}\right) \psi+G_{S} \sum_{a=0}^{8}\left[\left(\bar{\psi} \lambda^{a} \psi\right)^{2}+\left(\bar{\psi} i \gamma^{5} \lambda^{a} \psi\right)^{2}\right] \\
& -G_{D}\left(\operatorname{det}\left[\bar{\psi}\left(1+\gamma_{5}\right) \psi\right]+\operatorname{det}\left[\bar{\psi}\left(1-\gamma_{5}\right) \psi\right]\right) \\
& -G_{V} \sum_{a=0}^{8}\left[\left(\bar{\psi} \gamma^{\mu} \lambda^{a} \psi\right)^{2}+\left(\bar{\psi} \gamma^{\mu} \gamma_{5} \lambda^{a} \psi\right)^{2}\right] \\
& -\mathcal{U}(\Phi, \bar{\Phi} ; T) .
\end{aligned}
$$

Here the quark field is represented by $\psi=(u, d, s)^{T}$ in flavor space, and $\hat{m}=\operatorname{diag}_{f}\left(m_{u}, m_{d}, m_{s}\right)$ is the corresponding (current) diagonal mass matrix. Finite-density effects are included by considering a finite quark chemical potential matrix, $\hat{\mu}=\operatorname{diag}_{f}\left(\mu_{u}, \mu_{d}, \mu_{s}\right)$. The Lagrangian includes a scalar-pseudoscalar interaction which spontaneously breaks chiral symmetry in the vacuum by generating a quark-antiquark condensate. Also present is the so-called Kobayashi-Maskawa-'t Hooft interaction, responsible for the generation of six-fermion interactions [26,27] which explicitly break the $U_{A}(1)$ symmetry and correctly reproduce the observed hadron spectra. We also include in the model a vector interaction. This term includes both a vector-isoscalar and a vector-isovector interaction with a coupling constant $G_{V}$ [28].

Since we are not interested in fixing the value of this parameter by reproducing vector-meson masses, we will study the effect of having a finite ratio $\zeta=G_{V} / G_{S}$ which is known to be important in the study of neutron stars [18,23] or the QCD phase diagram [29]. Indeed, even fixing the vector interaction in the vacuum does not restrict its possible in-medium dependence since it couples to density degrees of freedom.

The PNJL model is also capable of describing the statistical confinement-deconfinement transition, with the breaking of $Z\left(N_{c}\right)$ symmetry. The quark fields are minimally coupled to a background gluonic field in the temporal direction, $A_{4}^{0}$, through the covariant derivative $D_{\mu}=\partial_{\mu}-A_{4}^{0} \delta_{\mu}^{0}$. An approximate order parameter for this transition is the Polyakov loop $\Phi$. In the confined phase $\Phi \rightarrow 0$, while in the deconfined phase $\Phi \rightarrow 1$. In this model, the effective potential $\mathcal{U}(\Phi, \bar{\Phi} ; T)$ is built using the Ginzburg-Landau theory of phase transitions: at low temperatures the $Z\left(N_{c}\right)$ symmetry holds, while at high temperatures it is broken. We choose to adopt the effective potential proposed in Refs. [30-32]:

$$
\begin{aligned}
\frac{\mathcal{U}(\Phi, \bar{\Phi} ; T)}{T^{4}}= & -\frac{1}{2} a(T) \bar{\Phi} \Phi \\
& +b(T) \ln \left[1-6 \Phi \bar{\Phi}+4\left(\Phi^{3}+\bar{\Phi}^{3}\right)-3(\Phi \bar{\Phi})^{2}\right],
\end{aligned}
$$

with the $T$-dependent parameters [32]

$a(T)=a_{0}+a_{1}\left(\frac{T_{0}}{T}\right)+a_{2}\left(\frac{T_{0}}{T}\right)^{2}, \quad b(T)=b_{3}\left(\frac{T_{0}}{T}\right)^{3}$. 
Its parametrization values are $a_{0}=3.51, a_{1}=-2.47$, $a_{2}=15.2$, and $b_{3}=-1.75$ [32] obtained with $T_{0}=$ $270 \mathrm{MeV}$ to reproduce the lattice QCD result. Due to the presence of quarks we will rescale the critical temperature to $T_{0}=210 \mathrm{MeV}$.

The PNJL model is nonrenormalizable and a regularization scheme has to be introduced to deal with nonconvergent integrals in the model. In the present work, the divergent ultraviolet sea-quark integrals are regularized by a sharp cutoff $\Lambda$ in three-momentum space. For the NJL model parametrization, we consider $\Lambda=602.3 \mathrm{MeV}$, $m_{u}=m_{d}=5.5 \mathrm{MeV}, m_{s}=140.7 \mathrm{MeV}, G_{S} \Lambda^{2}=1.835$, and $G_{D} \Lambda^{5}=12.36$ [33].

Our goal is to describe a multicomponent system in which the local concentrations of charges are kept fixed across a phase-coexistence region. Although we are discussing the phase transition within a three-component system composed of quarks $u, d$, and $s$, the results we present are defined by the first two alone. The onset of $s$ quarks occurs for much larger baryonic densities and chemical potentials than the ones shown in the following figures. This was discussed in Ref. [29] and it was shown that the $s$ quark affects the QCD phase diagram only for a baryonic chemical potential $\mu_{B} \gtrsim 1400 \mathrm{MeV}$.

Since we will be discussing the behavior of matter with a fixed charge fraction with zero strangeness density $\left(\rho_{s} / \rho_{B}=Y_{S}=0\right)$, the Gibbs free energy $G$ is given by [4]

$$
G=B \mu_{B}+Q \mu_{Q}=B \mu_{B Q},
$$

where $B$ and $Q$ are the baryon and charge number, respectively, and $\mu_{B Q}=\mu_{B}+Y_{Q} \mu_{Q}$ is the chemical potential which is kept constant during a phase transition at constant charge fraction [4] if the strangeness charge is zero. As shown in Ref. [4], the following interphase chemical equilibrium condition is imposed: $\mu_{B Q}^{I}=\mu_{B Q}^{I I}$, with the local Gibbs free energy per baryon $\mu_{B Q}^{i}=\mu_{B}^{i}+Y_{Q}^{i} \mu_{Q}^{i}$, where $i=I$ and $I I$ designates the phase with broken chiral symmetry and the chiral-symmetric phase, respectively.

\section{RESULTS}

In the following, we discuss the first-order phase transition associated with the restoration of chiral symmetry in asymmetric quark matter, both with and without vector interactions. This will be implemented by fixing the value of $Y_{Q}$ and by the conservation of baryon number $B$ and total net strangeness $S=0$. Due to its relevance in $\mathrm{HICs}$, namely, for $\mathrm{Au}-\mathrm{Au}$ or $\mathrm{Pb}-\mathrm{Pb}$ collisions, we will frequently choose the charge fraction $Y_{Q}=0.4$ and $Y_{S}=0$ [34]. Another interesting scenario for asymmetric quark matter is the one occurring in core-collapse supernova matter where matter has a proton fraction below 0.4. Scenarios with isospin charge conservation in strong interaction were also studied in Refs. [35-37]. Although strangeness may occur due to the weak interaction, in the following discussion we consider $Y_{S}=0$.

We first analyze the phase diagram of the PNJL model with $Y_{Q}=0.5$. This specific value for $Y_{Q}$ corresponds to describing one-fluid matter consisting of symmetric matter, with equal amount of quarks $u$ and $d\left(\rho_{u}=\rho_{d}, \rho_{s}=0\right)$, and, in this case, the phase transition is governed by $\mu_{B}$. The same concerns the study of pure neutron matter with charge fraction $Y_{Q}=0$, which we will also refer to in the following. To study the effect of a finite repulsive vector interaction, we will present results for models with $\zeta=$ $G_{V} / G_{S}=0$ and $\zeta=G_{V} / G_{S}=0.5$. The last value was chosen in order to have the critical end point (CEP) at $T \approx 0$ for $Y_{Q}=0$.

In Fig. 1 the phase diagram of the model is shown in terms of the baryonic chemical potential (left panel) and the baryonic density (right panel). In both panels the spinodal, binodal, and crossover lines are represented by black, thick red, and thin red lines, respectively, and the deconfinement crossover is represented by the blue lines. In the right panel, the regions between the binodal and spinodal lines and the region inside the spinodal section correspond to regions of metastable and unstable matter, respectively. They are reached only during a nonequilibrium evolution of the system. Under the present conditions, homogeneous chiralsymmetric quark matter is attained at densities above $0.4 \mathrm{fm}^{-3}$. However, below these densities chiral-symmetric quark matter will have the form of clusterized matter. One of the main objectives of the present study is to understand under which conditions chiral-symmetric quark matter is more favorably formed.

In the left panel of Fig. 2, we plot the pressure as a function of the baryon density to exemplify a phase transition from the broken phase to the symmetric one at $T=50 \mathrm{MeV}$, for $\zeta=0$ (curve $A B$, in red) and $\zeta=0.5$ (curve $A^{\prime} B^{\prime}$, in blue). This phase transition is obtained by keeping $Y_{Q}$ fixed during the transition; as a consequence, the pressure rises slightly. It also shows that the phase transition is weaker, i.e., corresponds to a smaller jump in density, when taking $\zeta=0.5$. This result is expected since the addition of the vector interaction is known to drag the first-order phase transition towards lower temperatures and make the pressure stiffer at smaller densities. For $\zeta>0.5$ and neutral matter with $Y_{Q}=0$, the first-order phase transition does not exist any more.

The right panel of Fig. 2 shows the phase diagram in the $T$-P plane for both $\zeta=0$ and $\zeta=0.5$, with the charge ratio $Y_{Q}=0.4$. As discussed before, the pressure is not constant in a phase transition with a charge fraction different from 0 or 0.5 . The bands define the range of pressures covered during the phase transition. When a finite repulsive vector interaction is considered the pressure at the transition increases considerably.

In Fig. 3 the phase transition lines at different charge fractions are plotted for $\zeta=0$ (left panels) and $\zeta=0.5$ 

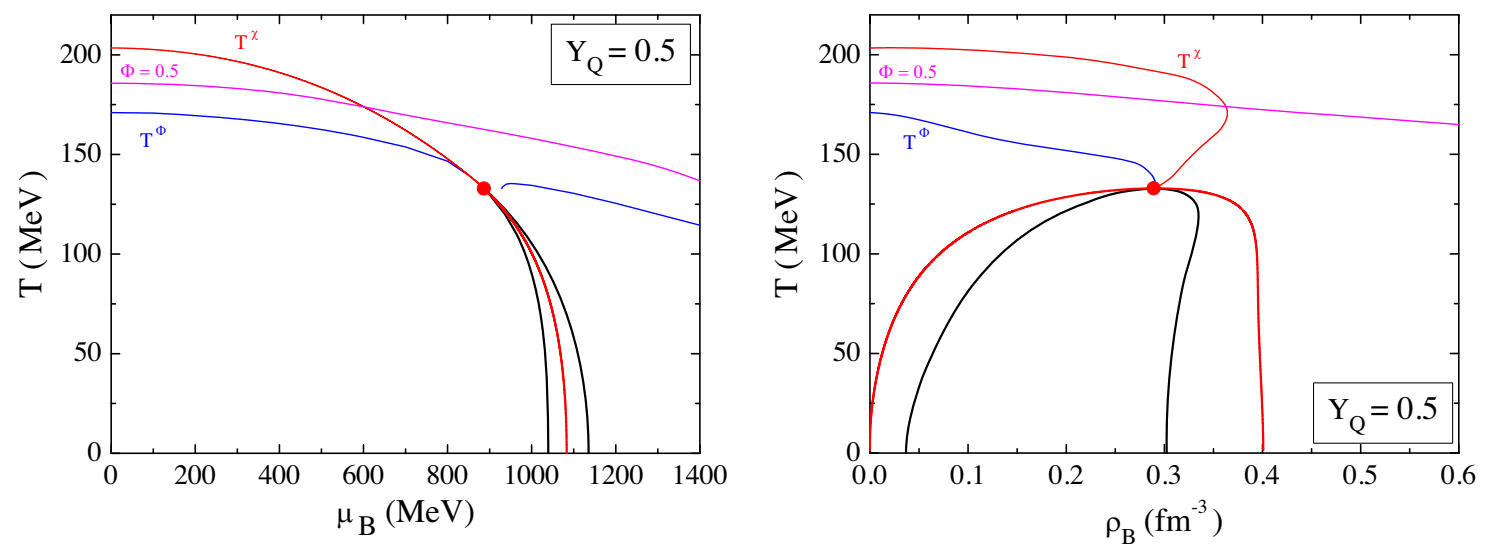

FIG. 1. Phase diagram of the PNJL model with $Y_{Q}=0.5$, in the $T-\mu_{B}$ (left) and $T-\rho_{B}$ (right) planes for the model with $T_{0}=210 \mathrm{MeV}$. The red lines are the crossover (thin lines) and first-order chiral transition (thick lines), the blue lines define the deconfinement crossover, and the purple lines represent $\Phi=0.5$. The black lines are the spinodal lines and the red dot is the CEP in this scenario.
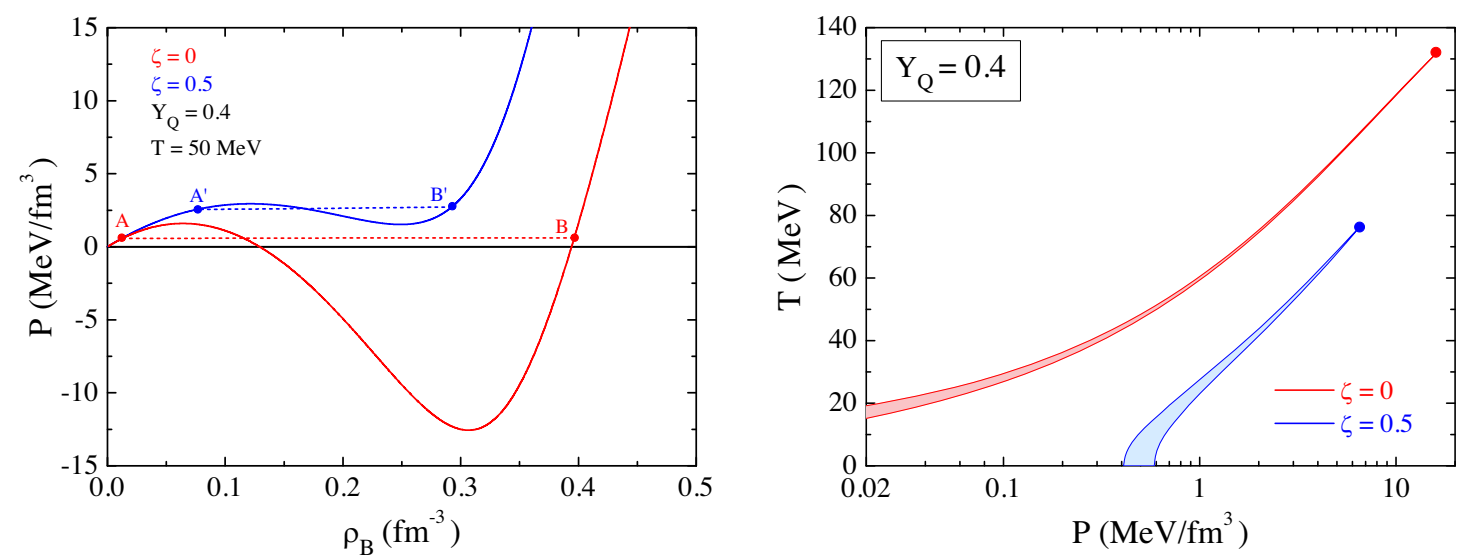

FIG. 2. Left: phase transition for asymmetric matter: pressure versus the baryonic density $\rho_{B}$ for $Y_{Q}=0.4, T=50 \mathrm{MeV}$, and $\zeta=G_{V} / G_{S}=0$ and $\zeta=G_{V} / G_{S}=0.5$. The phase transition is indicated with a thin dashed line. Since it is a two-component system the transition does not occur at constant pressure. The coordinates $\left(\rho_{B}, P\right)$ in $\left(\mathrm{fm}^{-3}, \mathrm{MeV} \mathrm{fm}^{-3}\right)$ of the different points shown in the plot are $A(0.013,0.565), B(0.397,0.613)$, and $A^{\prime}(0.076,2.56), B^{\prime}(0.292,2.73)$. Right: transition lines in the $T-P$ plane for $Y_{Q}=0.4$, with $\zeta=0$ and 0.5 .

(right panels) in the $T-\mu_{B}$ plane (upper panels) and $T-\rho_{B}$ plane (lower panels). The two extremes $Y_{Q}=0$ and $Y_{Q}=$ 0.5 describe one-component matter: neutral matter with zero total electric charge and symmetric matter with an equal amount of $u$ and $d$ quarks, respectively. In those extreme cases, identified in Fig. 3 by dashed lines, the phase transition occurs at constant pressure and baryonic chemical potential and also reduces to a line in the $T-\mu_{B}$ plane.

For any other given $Y_{Q}$ in the $T-\mu_{B}$ or $T-\rho_{B}$ plane, the line at which matter starts to recover chiral symmetry and the line for which matter is already in a partial chiralsymmetric state $^{1}$ are not coincident (see Fig. 3). However,

\footnotetext{
${ }^{1}$ A partial chiral-symmetric state in the sense that the constituent quark mass is still far from the respective current one.
}

in the $T-\mu_{B Q}$ plane the phase transition is defined by a single line.

The effect of including a finite vector interaction is clear: for a given $Y_{Q}$, a finite $\zeta$ pushes the critical region and CEP toward higher values of the chemical potential $\mu_{B}$ and lower values of the temperature. The main effect on the phase transition of a charge fraction smaller than 0.5 is seen in the localization of the CEP and the onset of the transition. In asymmetric matter, i.e., with a smaller total charge, the CEP moves to smaller temperatures and larger $\mu_{B}$ and the onset of the transition moves to larger values of $\mu_{B}$. For $\zeta=0$, the CEP chemical potential $\left(\mu_{B}^{\mathrm{CEP}}\right)$ suffers a rather large change with the reduction of the total charge $Y_{Q}$ : going from $Y_{Q}=0.5$ to $Y_{Q}=0, \mu_{B}^{\mathrm{CEP}}$ varies $\approx 89 \mathrm{MeV}$, while the temperature $T^{\mathrm{CEP}}$ is only moderately affected, suffering 

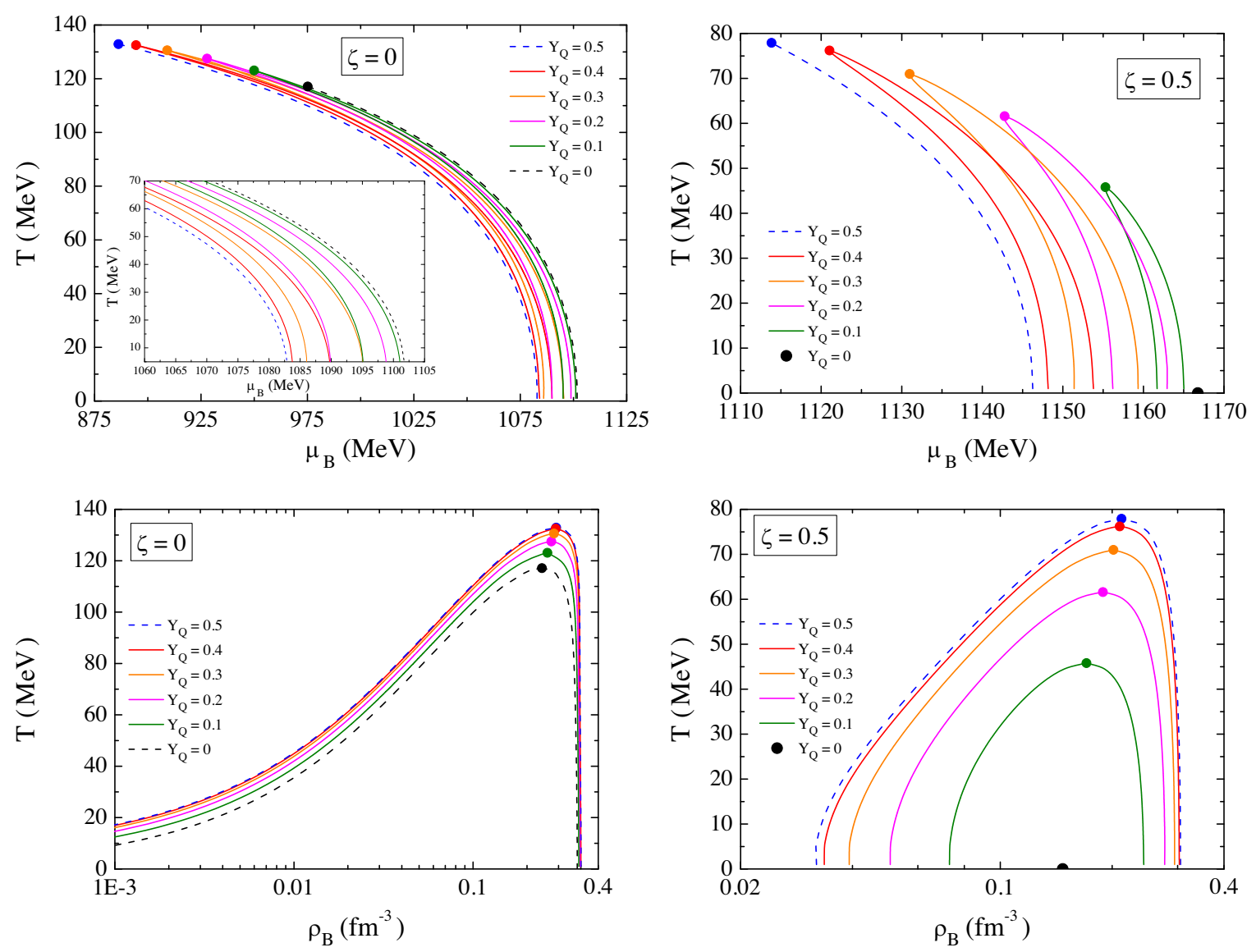

FIG. 3. Transition lines in the $T-\mu_{B}$ plane (top panels) and $T-\rho_{B}$ plane (bottom panels) for $\zeta=0$ (left panels) and $\zeta=0.5$ (right panels). The dots are the respective CEPs. In the two bottom panels a log scale is used on the $x$ axis.

a reduction of $\approx 16 \mathrm{MeV}$. If $\zeta=0.5$, the CEP temperature experiences the largest variation with decreasing $Y_{Q}$, with a reduction of $\approx 78 \mathrm{MeV}$, while the change in $\mu_{B Q}^{\mathrm{CEP}}$ is not larger than $54 \mathrm{MeV}$. In this case, the CEP occurs at zero temperature for $Y_{Q}=0$.

There is no first-order phase transition for $\zeta=0.5$ with $Y_{Q}=0$ because the CEP occurs at $T=0$. This is similar to neutron matter, for which there is no liquid-gas phase transition [5]. The possible existence of a first-order phase transition to a chirally symmetric state in neutral matter depends strongly on the coupling constant $G_{V}$, which plays a role similar to the one played by the $\rho$-meson coupling for nuclear relativistic mean-field models.

For all other charge fractions represented, the phase transition is defined by two lines in the $T-\mu_{B}$ plane; see both top panels of Fig. 3. The range of temperatures and chemical potentials spanned by the $\zeta=0$ model is much larger, but the overall features are similar. For each charge fraction, in the region between the left branch (at the lower chemical potential or density limit) and the right branch (at the larger chemical potential or density limit) matter separates into two phases: a low-density phase with broken chiral symmetry and a high-density (partially) chiral-symmetric matter.
In the bottom panels of Fig. 3, the transition lines are represented in the $T-\rho_{B}$ plane and the presence of the vector interaction has a noticeable effect. The line that defines the left border of the transition region occurs at very low densities if $\zeta=0$ and $T \lesssim 20 \mathrm{MeV}$. On the other extreme, all lines come close to $\rho_{B}=0.4 \mathrm{fm}^{-3}$, which lies below 3 times the saturation density. The smaller the charge fraction, the lower the critical temperature, the smaller the transition region, and the smaller the density at which chiral-symmetric matter sets in, although the differences are not very large if $\zeta=0$. A different situation occurs for $\zeta=0.5$; in this case, both the critical temperature and the width of the transition decrease drastically with decreasing $Y_{Q}$, and at $Y_{Q}=0$ the phase transition is reduced to a point (the CEP).

An important conclusion is that for a finite $\zeta$ the transition to chiral-symmetric matter may occur at quite low densities, making this phase more accessible experimentally. For matter characterized by $Y_{Q}=0.4$ this is possible already at twice saturation density for a small temperature, and at an even smaller density for larger temperatures. If during a nonequilibrium reaction the system enters the region inside the binodal or spinodal, the density for the appearance of chiral symmetric matter 

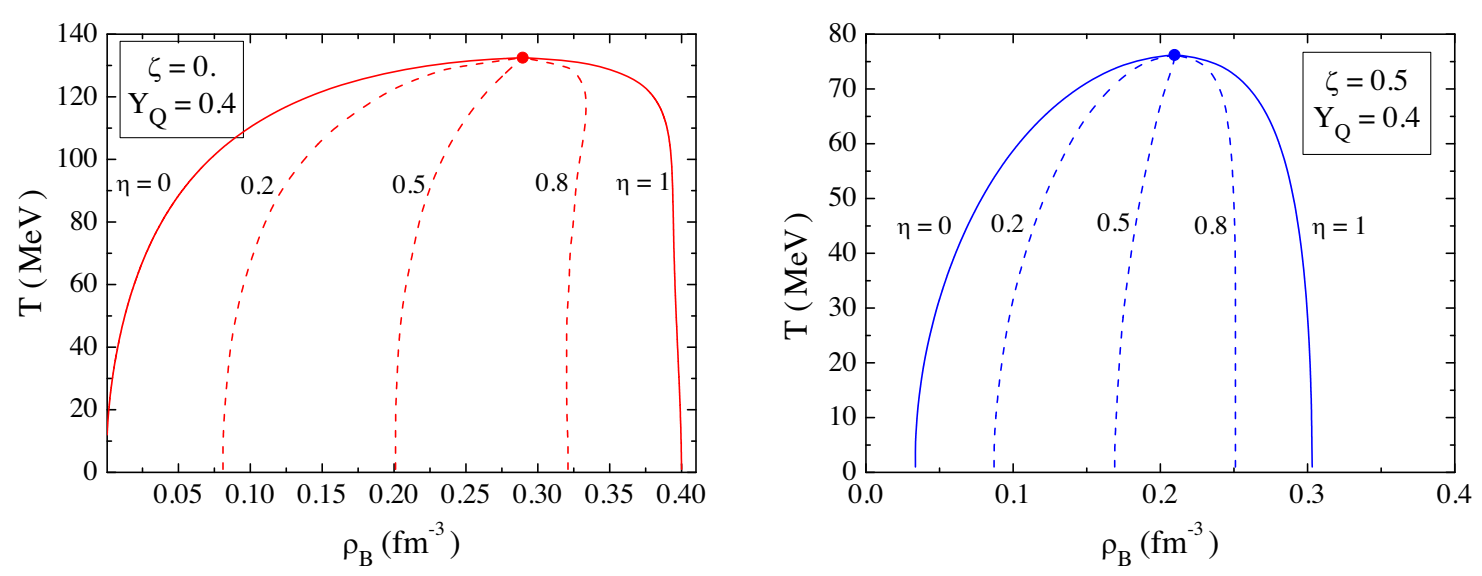

FIG. 4. The transition lines (full lines) in the $T-\rho_{B}$ plane for $\zeta=0$ (left) and $\zeta=0.5$ (right): the left curve corresponds to matter in a chiral-symmetric broken state and the right curve to matter in a chiral-symmetric state. The dashed lines identify the localization matter where $20 \%, 50 \%$, and $80 \%$ of the matter is in a chiral-symmetric state, respectively.

will be even lower as we will discuss in the following. In this case, however, in the form of clusterized matter. For $\zeta=0$ the unstable/metastable region extends until very low densities, while for $\zeta=0.5$ the low-density limit of the mixed phase sets in between 0.01 and $01 \mathrm{fm}^{-3}$ depending on the charge.

In Fig. 4 the phase diagrams in the $T-\rho_{B}$ plane are shown for $Y_{Q}=0.4$, with $\zeta=0$ (left panel) and $\zeta=0.5$ (right panel). The full lines identify the limits of the mixed phase: $\eta=0$ means that the matter is all in a broken-chiralsymmetry phase, while matter with $\eta=1$ is in a completely restored-chiral-symmetry (partially) phase. Between the limiting lines of the mixed phase, several dashed lines show where matter is constituted by different fractions of chiralsymmetric matter, identified by $\eta$. Within the models we have considered, it is possible to form chiral-symmetric matter in clusterized matter at densities well below the onset of pure chiral-symmetric matter, during a nonequilibrium evolution of the system.

\section{CONCLUSIONS}

In this work we studied how the restoration of chiral symmetry, within the PNJL model with and without vector interactions, is affected by the conservation of more then one charge. Although a three-flavor quark model was considered, the critical region studied is only defined by the up and down quarks, because the effect of the $s$ quarks is only felt at higher energies than the ones considered.

During a phase transition involving more than one conserved charge, pressure is not constant, indicating the presence of a mixed phase. In this case, the transition region defined in the $T-\mu_{B}$ or $T-\rho_{B}$ plane is limited by two distinct lines, divided by a mixed phase. The width of this mixed phase is dependent on the total charge fraction $Y_{Q}$ and heavily dependent on the vector coupling $\zeta=G_{V} / G_{S}$.

The inclusion of a vector interaction weakens the phase transition both when considering a one-fluid system (such as those consisting of symmetric matter or neutral matter) or a system with more than one conserved charge (such as asymmetric quark matter with a charge fraction $0<Y_{Q}<0.5$ ). In particular, when taking a sufficiently strong vector coupling the CEP disappears.

It was shown that for asymmetric matter the restoration of chiral symmetry occurs at smaller densities, as suggested in Ref. [38], and that the binodal section is reduced; in particular, the low-density onset and high-density limits of the binodal move to larger and smaller densities, respectively. This effect is stronger if the vector interaction is included. Besides, the localization of the CEP also moves to lower temperatures.

During a nonequilibrium evolution, the system may reach regions of the phase diagram, such as the metastable or unstable regions inside the binodal and spinodal sections, that are forbidden to equilibrium thermodynamics [24]. We have shown that under these conditions it is possible to form clusters made of chiral-symmetric matter at rather low densities. It is clear that these densities are model dependent. However, the fact that asymmetric matter favors the formation of chiral-symmetric matter at lower densities than the predictions from symmetric matter is quite general. These results are, in particular, of interest for neutron stars where the proton fraction is especially low. Besides, it was also shown that the occurrence of a quark phase inside two solar-mass neutron stars requires the inclusion of the vector interaction in the Lagrangian density of NJL-like models; see, for instance, Refs. [18,23,39]. These two characteristics - the asymmetry of matter and the presence of the vector interaction-may indicate that in hot environments (such as in neutron star mergers or protoneutron stars) the presence of chiral-symmetric quark matter is possible even at moderate densities. Carrying these conclusions to the laboratory, they seem to show that using large neutron-rich nuclei in heavy collisions may give rise to conditions favorable to the formation of clusters of 
chiral-symmetric quark matter at intermediate densities attainable, for instance, at FAIR or NICA. Even stable heavy nuclei such as lead or uranium with a charge fraction $\sim 0.4$ would already be sufficiently asymmetric to create the necessary conditions for quark matter formation. Unstable radioactive beams of very asymmetric heavy nuclei would further improve these conditions.

In the present study we have paid special attention to conditions presently attained at the RHIC, in particular, a charge fraction of 0.4 (as in $\mathrm{Pb}-\mathrm{Pb}$ collisions) and zero strangeness fraction, i.e., the strangeness density is put to zero. In order to connect our results with lattice QCD calculations, we have checked the leading-order contribution in $\mu_{B}$ for the expansions of the electric charge and strangeness chemical potentials, $\mu_{Q}$ and $\mu_{S}$ (see Refs. [40,41]). For chemical potentials below $400 \mathrm{MeV}$ and temperatures of the order of $200 \mathrm{MeV}$, these temperature-dependent coefficients are $q_{1}=-0.04$ and $s_{1}=0.34$. A more extended comparison with lattice QCD data, within a larger region of temperature and chemical potentials, is beyond the scope of this work and is left for upcoming investigations.

As future work, it would be interesting to study this scenario in a QCD model beyond the mean-field approximation. One way to incorporate quantum fluctuations in this calculation is by using the functional renormalization group (FRG). Recently, the QCD phase has been studied by applying the FRG approach to the quark meson model [42-46]. The influence of strangeness neutrality on thermodynamic quantities such as the equation of state was studied in Ref. [47]. Imposing strangeness conservation and the initial isospin asymmetry of the colliding nuclei is the natural step forward to continue these studies.

\section{ACKNOWLEDGMENTS}

This work was supported by national funds from FCT (Fundação para a Ciência e a Tecnologia, I.P, Portugal) under the IDPASC Ph.D. program (International Doctorate Network in Particle Physics, Astrophysics and Cosmology), with the Grant No. PD/BD/128234/2016 (R. C. P.), and under the Projects No. UID/FIS/04564/2019, No. UID/04564/ 2020, and No. POCI-01-0145-FEDER-029912 with financial support from POCI "Programa Operacional Competitividade e Internacionalização (COMPETE 2020)," in its FEDER component. Partial support comes from "THOR" (COST Action CA15213) and "PHAROS" (COST Action CA16214).
[1] C. Greiner, P. Koch, and H. Stoecker, Phys. Rev. Lett. 58, 1825 (1987).

[2] N. K. Glendenning, Phys. Rev. D 46, 1274 (1992).

[3] N. K. Glendenning, Compact Stars: Nuclear Physics, Particle Physics, and General Relativity (Springer-Verlag, New York, 1997).

[4] M. Hempel, V. Dexheimer, S. Schramm, and I. Iosilevskiy, Phys. Rev. C 88, 014906 (2013).

[5] H. Muller and B. D. Serot, Phys. Rev. C 52, 2072 (1995).

[6] C. Ducoin, P. Chomaz, and F. Gulminelli, Nucl. Phys. A771, 68 (2006).

[7] W.-j. Fu, Z. Zhang, and Y.-x. Liu, Phys. Rev. D 77, 014006 (2008).

[8] M. Ciminale, R. Gatto, N. D. Ippolito, G. Nardulli, and M. Ruggieri, Phys. Rev. D 77, 054023 (2008).

[9] K. Fukushima, Phys. Rev. D 77, 114028 (2008); 78, 039902(E) (2008).

[10] H. Abuki, R. Anglani, R. Gatto, G. Nardulli, and M. Ruggieri, Phys. Rev. D 78, 034034 (2008).

[11] P. Costa, M. C. Ruivo, C. A. de Sousa, and H. Hansen, Symmetry 2, 1338 (2010).

[12] M. Asakawa and K. Yazaki, Nucl. Phys. A504, 668 (1989).

[13] N. M. Bratovic, T. Hatsuda, and W. Weise, Phys. Lett. B 719, 131 (2013).

[14] J. Braun, M. Leonhardt, and M. Pospiech, Phys. Rev. D 97, 076010 (2018).
[15] J. Braun, M. Leonhardt, and M. Pospiech, Phys. Rev. D 96, 076003 (2017).

[16] M. F. Lutz, S. Klimt, and W. Weise, Nucl. Phys. A542, 521 (1992).

[17] M. Hanauske, L. Satarov, I. Mishustin, H. Stoecker, and W. Greiner, Phys. Rev. D 64, 043005 (2001).

[18] L. Bonanno and A. Sedrakian, Astron. Astrophys. 539, A16 (2012).

[19] D. Logoteta, C. Providência, and I. Vidaña, Phys. Rev. C 88, 055802 (2013).

[20] T. Beisitzer, R. Stiele, and J. Schaffner-Bielich, Phys. Rev. D 90, 085001 (2014).

[21] A. Zacchi, R. Stiele, and J. Schaffner-Bielich, Phys. Rev. D 92, 045022 (2015).

[22] P. Costa, Phys. Rev. D 93, 114035 (2016).

[23] R. Câmara Pereira, P. Costa, and C. Providência, Phys. Rev. D 94, 094001 (2016).

[24] C. Sasaki, B. Friman, and K. Redlich, Phys. Rev. Lett. 99, 232301 (2007).

[25] C. Sasaki, B. Friman, and K. Redlich, Phys. Rev. D 77, 034024 (2008).

[26] S. P. Klevansky, Rev. Mod. Phys. 64, 649 (1992).

[27] T. Hatsuda and T. Kunihiro, Phys. Rep. 247, 221 (1994).

[28] I. N. Mishustin, L. M. Satarov, H. Stoecker, and W. Greiner, Phys. Rev. C 62, 034901 (2000).

[29] M. Ferreira, P. Costa, and C. Providência, Phys. Rev. D 98, 034006 (2018). 
[30] K. Fukushima, Phys. Lett. B 591, 277 (2004).

[31] C. Ratti, M. A. Thaler, and W. Weise, Phys. Rev. D 73, 014019 (2006).

[32] S. Roessner, C. Ratti, and W. Weise, Phys. Rev. D 75, 034007 (2007).

[33] P. Rehberg, S. P. Klevansky, and J. Hufner, Phys. Rev. C 53, 410 (1996).

[34] A. N. Sissakian, A. S. Sorin, and V. D. Toneev, Conf. Proc. C 060726, 421 (2006).

[35] R. Cavagnoli, C. Providencia, and D. P. Menezes, Phys. Rev. C 83, 045201 (2011).

[36] G. Y. Shao, M. Di Toro, V. Greco, M. Colonna, S. Plumari, B. Liu, and Y. X. Liu, Phys. Rev. D 84, 034028 (2011).

[37] G. Y. Shao, M. Colonna, M. Di Toro, B. Liu, and F. Matera, Phys. Rev. D 85, 114017 (2012).

[38] N. Kaiser and W. Weise, Phys. Lett. B 671, 25 (2009).
[39] S. Benic, D. Blaschke, D. E. Alvarez-Castillo, T. Fischer, and S. Typel, Astron. Astrophys. 577, A40 (2015).

[40] A. Bazavov et al., Phys. Rev. Lett. 109, 192302 (2012).

[41] S. Borsanyi, Z. Fodor, S. Katz, S. Krieg, C. Ratti, and K. Szabo, Phys. Rev. Lett. 111, 062005 (2013).

[42] B.-J. Schaefer and J. Wambach, Nucl. Phys. A757, 479 (2005).

[43] T. K. Herbst, J. M. Pawlowski, and B.-J. Schaefer, Phys. Rev. D 88, 014007 (2013).

[44] R.-A. Tripolt, N. Strodthoff, L. von Smekal, and J. Wambach, Phys. Rev. D 89, 034010 (2014).

[45] R.-A. Tripolt, B.-J. Schaefer, L. von Smekal, and J. Wambach, Phys. Rev. D 97, 034022 (2018).

[46] R. Câmara Pereira, R. Stiele, and P. Costa, Eur. Phys. J. C 80, 712 (2020).

[47] W.-j. Fu, J. M. Pawlowski, and F. Rennecke, SciPost Phys. Core 2, 002 (2020). 